\title{
Hole-Induced Electron Transport through Core-Shell Quantum Dots: A Direct Measurement of the Electron-Hole Interaction
}

\author{
Ingmar Swart, ${ }^{*}{ }^{\dagger}{ }^{\ddagger}$ Zhixiang Sun, ${ }^{\dagger}$ Daniël Vanmaekelbergh, ${ }^{\dagger}$ and Peter Liljeroth*, ${ }^{*} \S$ \\ ${ }^{\dagger}$ Condensed Matter and Interfaces, Debye Institute for Nanomaterials Science, University of Utrecht, P.O. Box \\ 80000, 3508 TA Utrecht, The Netherlands, ${ }^{\ddagger}$ Institute for Experimental and Applied Physics, Faculty of Physics, \\ University of Regensburg, Universitätsstrasse 31, D-93053, Regensburg, Germany, and ${ }^{\S}$ Low Temperature \\ Laboratory, Aalto University, PO Box 15100, FI-00076 AALTO, Finland
}

\begin{abstract}
Quantum dots (QDs) have promising optoelectronic properties. Colloidal QD heterostructures, systems in which two semiconductors are incorporated in a single colloid, may show novel and potentially useful transport phenomena. Here, we report on the physical mechanisms of charge transport through PbSe-CdSe core-shell QDs measured with cryogenic scanning tunneling spectroscopy. Compared to single-component QDs, an additional hole-induced electron tunneling channel is found. Electron tunneling with and without a hole occurs at different bias, allowing the determination of the electron-hole interaction energy ( 80 meV). This energy is sufficiently large to allow for a transport regime at room temperature in which electrons tunnel into the dot only if a hole is present, an ideal situation for controlled single-photon emission.
\end{abstract}

KEYWORDS Core-shell quantum dots, scanning tunneling microscopy, scanning tunneling spectroscopy, single-electron tunneling, electron-hole interaction, ambipolar electron transport

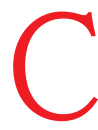
olloidal semiconductor quantum dots (QDs) are wellknown for their tunable optoelectronic properties; for example, their emission color can be adjusted by changing the QD size due to quantum confinement. ${ }^{1-3}$ Recent advances in the wet-chemical synthesis of QDs have further enhanced these possibilities by making it possible to create heteronanostructures, where two different semiconductors are incorporated into a single colloidal QD. ${ }^{4-9}$ As the band offsets can be tuned through the choice of the materials and by quantum confinement, the optoelectronic properties of these materials can be engineered with a high level of control. ${ }^{2,4,5}$ In particular, the resulting systems can be classified according to their relative band alignment (see Figure 1a). "Type I" is where both the electrons and holes are confined in the core or "type II" where electrons and holes are separated. ${ }^{2,4}$ Type II structures have recently attracted considerable interest as the formation of indirect excitons (separation of the electron-hole pair between the core and the shell) leads to significant increase in the exciton lifetime with possible applications in, for example, solar cells and zero-threshold lasers. ${ }^{9,10}$

The heteronanostructures have been previously studied by optical spectroscopy focusing on the effects of band alignment on absorption and emission spectra and exciton

* To whom correspondence should be addressed. E-mail: (I.S.) ingmar.swart@ physik.uni-regensburg.de; (P.L.) p.liljeroth@uu.nl.

Received for review: 03/17/2010

Published on Web: 04/14/2010 recombination dynamics. On the other hand, heteronanocrystals may show novel charge transport phenomena related to the asymmetric transport pathways for electrons and holes. This should have important consequences for opto-
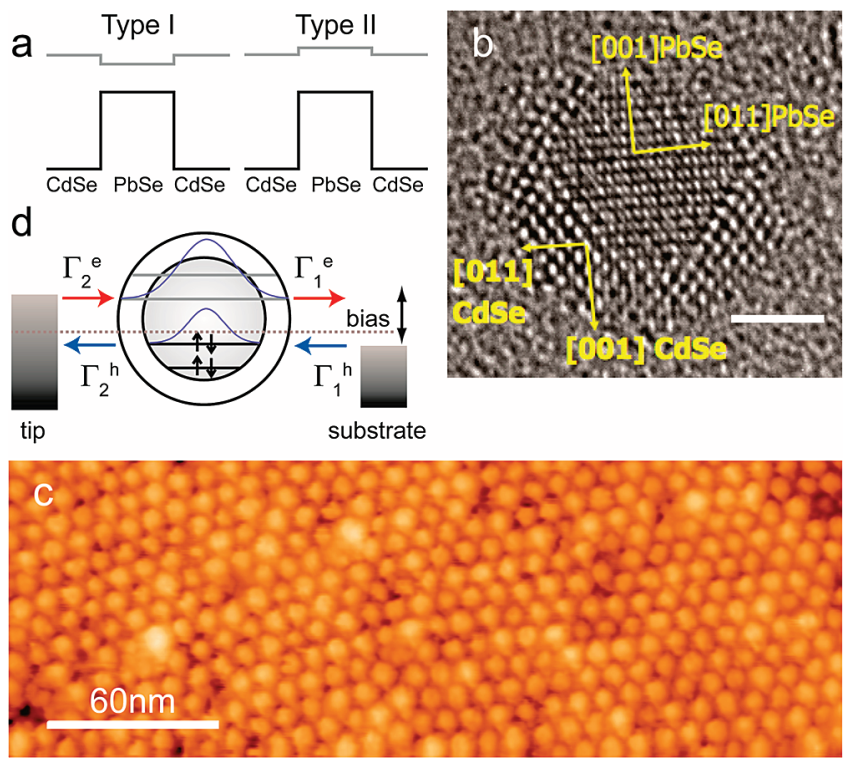

FIGURE 1. (a) Possible band-alignments of PbSe-CdSe core-shell QDs. (b) High-resolution TEM image of a PbSe-CdSe core-shell QD. Orientations of different crystal planes are indicated. Scale bar: 2 $\mathrm{nm}$. (c) STM topography image of an array of PbSe-CdSe QDs on an HOPG substrate. (d) Schematic illustration of the transport processes in a double-barrier tunnel junction. 
electronic devices that convert light into electrical current, or vice versa. The effects on charge transport due to the quantization of charge and the discrete energy levels of nanostructures are well-established. ${ }^{11-14}$ For instance, addition of a single electron is possible only if the associated energy barrier is overcome, for example, by applying a sufficient bias voltage between the source and the drain electrodes (Coulomb blockade). It should be realized, however, that in devices where both electrons and holes are present, new effects arise due to the electron-hole attraction and possibly, spin interactions. ${ }^{14,15}$ Understanding these effects requires well-defined, quantitative experiments that allows one to separate the electron and hole currents and measure the electron-hole interaction energy. Since optical experiments inherently involve the creation or annihilation of both an electron and a hole simultaneously, the electronhole interaction cannot be measured by optical means.

Under ambipolar charge transport conditions the electron-hole attraction will lower the energy barrier associated with electron tunneling. The offset between the hole-induced and bare electron tunneling channels provides a direct experimental access to the electron-hole interaction energy (vide infra). However, hole-induced electron tunneling is usually obscured by the fact that it is impossible to separate electron and hole currents and, hence, direct measurements of the electron-hole interaction energy have not been reported. In addition, ambipolar transport typically requires high bias voltages. ${ }^{16-18}$ Here, we demonstrate that these complications can be avoided by using PbSe-CdSe core-shell QDs (Figure 1b), where the hole levels are localized in the PbSe core while the electron levels are delocalized over the entire heteronanostructure. This results from both the high effective mass of the hole in CdSe and the asymmetric band alignment between the conduction and valence bands of $\mathrm{PbSe}$ and CdSe. This type II (or quasi type II) band level alignment shown in Figure $1 \mathrm{a}$ allows holes to be injected into the core at low bias voltage, which gives rise to hole-induced electron tunneling, while simultaneously the absolute magnitude of the hole current is strongly suppressed due to the higher barrier and higher effective mass of holes in CdSe. Our experiments clearly demonstrate the simultaneous presence of two different tunneling channels, normal singleelectron tunneling and hole-induced single-electron tunneling. The peak-spacing gives thus direct information on the electron-hole interaction energy.

The charge transport through these core-shell QDs was investigated using low-temperature scanning tunneling microscopy (STM) and spectroscopy (STS), which have been extensively utilized in measuring the local density of states in single-electron charge transport experiments in QDs and QD assemblies. ${ }^{19-23}$ Colloidal PbSe-CdSe core-shell QDs (overall diameter $6 \mathrm{~nm}$ with a shell thickness of $\sim 1.5 \mathrm{~nm}$, Figure $1 \mathrm{~b}$ ) were synthesized through a cation-exchange reaction. ${ }^{24,25}$ and deposited on highly oriented pyrolytic graphite (HOPG) substrates as described previously. ${ }^{22,26}$
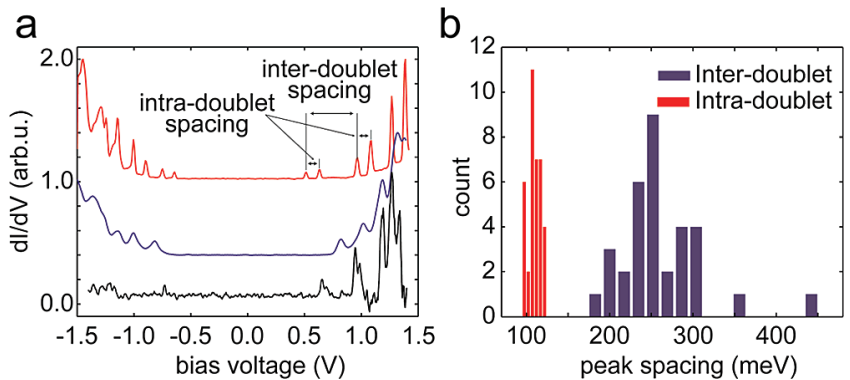

FIGURE 2. (a) Differential conductance spectra of a PbSe-CdSe core-shell QD (top), a $4.3 \mathrm{~nm}$ PbSe QD (middle), and a $6.1 \mathrm{~nm}$ CdSe QD (bottom). (b) Histogram of inter- and intradoublet peak spacing.

Electron microscopy investigations showed that the shellthickness is not isotropic within a QD, that is, there are regions with thicker and thinner shells. STM experiments (LT STM, Omicron Nanotechnology) were carried out at $T=5$ $\mathrm{K}$ with cut PtIr tips. A large scale STM image is shown in Figure 1c. The QDs appear as bright protrusions (bias voltage, $2.5 \mathrm{~V}$; set-point current, $10 \mathrm{pA}$ ). The differential conductance was recorded using a lock-in amplifier (amplitude $3 \mathrm{mV}$ rms at a frequency of $300 \mathrm{~Hz}$ ).

The overall band alignment of the PbSe-CdSe core-shell QDs is such that the hole-levels are strongly confined to the PbSe core, whereas the electron levels are more delocalized over the entire QD (Figure 1a). The tip-QD-substrate geometry forms a double-barrier tunnel junction, as shown in Figure $1 \mathrm{~d}$. It is characterized by the electron tunneling rates $\Gamma_{1}{ }^{\mathrm{e}}$ and $\Gamma_{2}{ }^{\mathrm{e}}$ and the hole tunneling rates $\Gamma_{1}{ }^{\mathrm{h}}$ and $\Gamma_{2}{ }^{\mathrm{h}}$, where the indices 1 and 2 refer to the tunneling barriers as defined in Figure $1 \mathrm{~d}$. The ratio $\Gamma_{1} \mathrm{e} / \Gamma_{2}{ }^{\mathrm{e}},\left(\Gamma_{1}{ }^{\mathrm{h}} / \Gamma_{2}{ }^{\mathrm{h}}\right)$ will determine the number of additional electrons (holes) in the QD. For example, in the limiting case $\Gamma_{1}{ }^{\mathrm{e}} \gg \Gamma_{2}{ }^{\mathrm{e}}$, that is, under shelltunneling conditions for electrons, electrons tunnel through the QD one by one, and $\mathrm{e}-\mathrm{e}$ interactions do not occur. In this case, the peaks in the tunneling spectrum at positive bias reflect the single-electron energy levels, provided that no holes are present.

A typical tunneling spectrum ( $\mathrm{d} I / \mathrm{d} V$ vs $V$ ) recorded on a PbSe-CdSe core-shell QD (top trace, $4.5 \mathrm{~nm}$ core, overall diameter $6 \mathrm{~nm}$ ) is compared to representative spectra of pure PbSe and CdSe QDs (middle and bottom traces, respectively) in Figure 2a. The spectrum of the core-shell $\mathrm{QD}$ is very different from those measured on pure PbSe and CdSe QDs, ${ }^{16,26,27}$ suggesting a novel electron transport mechanism through these heteronanostructures. The most striking observation is that the spectrum of the heterostructure consists of a series of doublet peaks with constant intradoublet spacing, absent in the spectra of pure CdSe or PbSe QDs. The interdoublet spacing is not constant, suggesting a different physical origin from the intradoublet spacing. We have observed these characteristic features for a significant fraction ( $30 \%$ ) of the core-shell QDs. Since the thickness of the CdSe shell is not isotropic (see Figure 
1b), the hole tunneling rates crucially dependent on the orientation of the QD on the substrate. Therefore it is likely that the position of the core in the tip-QD (core-shell)substrate junction is similar for all these QDs. In Figure 2b, a histogram of the measured peak-spacings is given for 20 QDs. The intradoublet spacing is strongly peaked around $110 \pm 8 \mathrm{meV}$, demonstrating that the spacing is essentially the same for all QDs. In contrast, the interdoublet spacing is considerably larger than the intradoublet spacing and is QD dependent. All the above strongly suggests that the doublets arise due to a charge transport mechanism that is specific to the PbSe-CdSe core-shell QDs.

To investigate the origin of the doublet-peaks, we performed simulations of the tunneling conductance spectra based on a master-equation approach, which is widely used in simulating electron and hole transport. ${ }^{11,12,14,18}$ Because of the band alignment in the PbSe-CdSe core-shell QDs, both the height and width of the tunneling barriers are larger for holes than for electrons (hole states are localized in the core, see Figure $1 \mathrm{~d}$ ). Hence, the hole-tunneling rates are smaller than the electron-tunneling rates. Since the experiments are performed under shell-tunneling conditions, the current is limited by the electron-tunneling rate from tip to $\mathrm{QD}, \Gamma_{2}{ }^{\mathrm{e}}$. This rate is set to $10^{8} \mathrm{~s}^{-1}$, as this gives tunneling currents comparable to the experimental set-point currents. Both electron and hole transport, as well as electron-hole recombination rate $(R)$ are considered. While we expect the electron tunneling to occur under shell-tunneling conditions, this may not hold for the holes. Because of the asymmetric position of the PbSe core in the QDs and the random deposition process, there is a distribution of different $\mathrm{QD}$ orientations in the arrays. The position of the core inside the QD strongly influences the widths of the tunneling barriers for holes, and therefore the hole-tunneling rates. Hence, a distribution in the $\Gamma_{2}{ }^{\mathrm{h}} / \Gamma_{1}{ }^{\mathrm{h}}$ ratios, and therefore in the observed spectra, can be expected. We will investigate this effect theoretically by varying the ratio of the hole-tunneling rates $\Gamma_{2}{ }^{\mathrm{h}} / \Gamma_{1}^{\mathrm{h}}$, while keeping the absolute values smaller than the electron-tunneling rates. The e-e interaction energy, $E_{\mathrm{e}}$ e, the position of the Fermi-level of the tip and substrate with respect to the $\mathrm{QD}$ energy levels, $\varepsilon_{\mathrm{F}}$, as well as the fraction of the applied bias that drops in the tip-QD junction, $\eta$, are considered as parameters. The polarization energies for electrons and holes, $E_{\mathrm{pol}}{ }^{\mathrm{e}}$ and $E_{\mathrm{pol}}{ }^{\mathrm{h}}$, are set to $E_{\mathrm{e}-\mathrm{e}} / 2 .{ }^{14,28}$

On the basis of our master equation simulations, reproducing the doublets observed experimentally requires that the Fermi levels of the tip and substrate are closer to the valence levels than to the conduction levels (at zero applied bias). In Figure $3 a$, the evolution of the simulated differential conductance spectra as a function of $\Gamma_{2}{ }^{\mathrm{h}}\left(10^{7}, 10^{6}, 10^{5}, 10^{4}\right.$, and $\left.10^{3} \mathrm{~s}^{-1}\right)$ is shown. $\Gamma_{1}{ }^{\mathrm{h}}$ was fixed at $10^{5} \mathrm{~s}^{-1}$. The hole energy level positions are such that hole injection occurs at positive bias before electron injection. Under the condition that $\Gamma_{2}{ }^{\mathrm{h}} \gg \Gamma_{1}{ }^{\mathrm{h}}$ (top trace in Figure 3a), all holes that tunnel into the $\mathrm{QD}$ at positive bias immediately tunnel out, leading

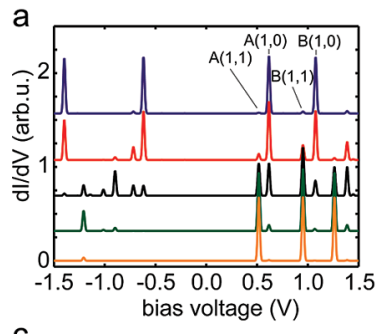

C
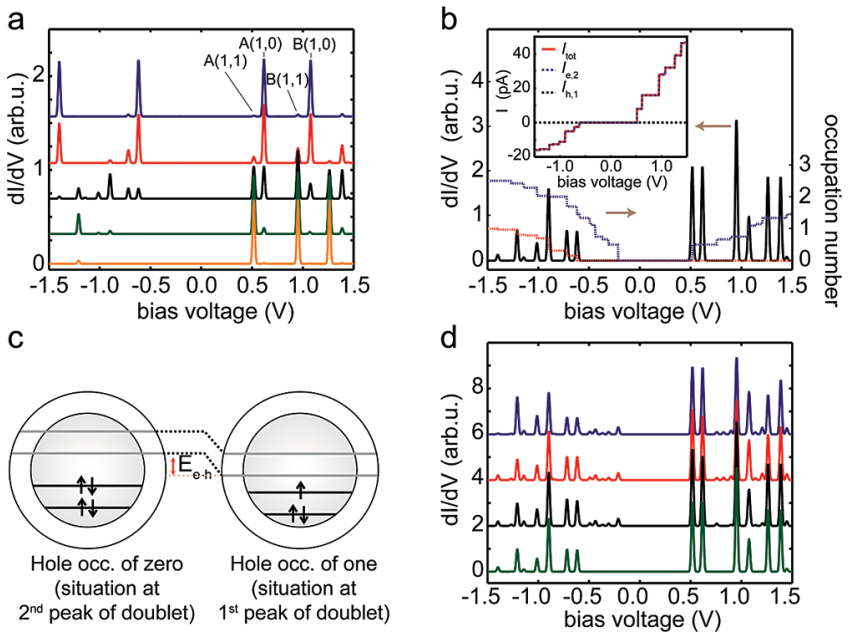

FIGURE 3. (a) Evolution of simulated differential conductance spectra as a function of $\Gamma_{2}^{\mathrm{h}}\left(10^{7}, 10^{6}, 10^{5}, 10^{4}\right.$, and $10^{3} \mathrm{~s}^{-1}$, bottom to top). $\Gamma_{1}{ }^{\mathrm{h}}$ was fixed at $10^{5} \mathrm{~s}^{-1}$. The labels $Z(x, y)$ refer to the conduction level, the number of holes and the number of electrons involved in the tunneling process, respectively. (b) Electron (red) and hole (blue) populations corresponding to $\Gamma_{2}{ }^{\mathrm{h}} / \Gamma_{1}{ }^{\mathrm{h}}=1$ (black spectrum, reproduced from figure $3 a$ ). The inset shows the relative contributions of electrons and holes to the total current. (c) Schematic of the influence of a hole on the electron levels. (d) Simulated differential conductance spectra, calculated with different values of the hole and electron tunneling rates. Parameter values given in Table 1.

TABLE 1. Parameter Values Used in the Calculation of the Differential Conductance Spectra Shown in Figure 3d

\begin{tabular}{lccccc}
\hline & $\Gamma_{1}{ }^{\mathrm{e}}\left(\mathrm{s}^{-1}\right)$ & $\Gamma_{2}{ }^{\mathrm{e}}\left(\mathrm{s}^{-1}\right)$ & $\Gamma_{1}{ }^{\mathrm{h}}\left(\mathrm{s}^{-1}\right)$ & $\Gamma_{2}{ }^{\mathrm{h}}\left(\mathrm{s}^{-1}\right)$ & $R\left(\mathrm{~s}^{-1}\right)$ \\
\hline blue spectrum & $10^{9}$ & $10^{8}$ & $10^{7}$ & $10^{7}$ & $10^{7}$ \\
red spectrum & $10^{9}$ & $10^{8}$ & $10^{7}$ & $10^{7}$ & 0 \\
black spectrum & $10^{12}$ & $10^{8}$ & $10^{7}$ & $10^{7}$ & 0 \\
green spectrum & $10^{12}$ & $10^{8}$ & $10^{0}$ & $10^{0}$ & 0
\end{tabular}

to an average hole occupation of zero. In this regime, the normal electron-tunneling resonance is observed. When $\Gamma_{2}{ }^{\mathrm{h}}$ $\ll \Gamma_{1}^{\mathrm{h}}$ (bottom trace in Figure 3a) electrons will always tunnel in the presence of a hole. In this situation, the nonzero hole occupation of the QD leads to an overall shift of the orbitals to lower energy (Figure 3c), causing the electron-tunneling channels to open at lower applied bias.

In Figure $3 b$, the spectrum simulated with $\Gamma_{2}{ }^{\mathrm{h}} / \Gamma_{1}{ }^{\mathrm{h}}=1$ is shown, together with the bias dependence of the electron and hole occupations calculated with the same parameters. For this ratio of $\Gamma_{2}{ }^{\mathrm{h}} / \Gamma_{1}{ }^{\mathrm{h}}$, the average hole occupation is smaller than the maximum value and therefore both the hole-induced electron tunneling channel and the conventional tunneling process occur. In this regime, the spectrum consists of a series of doublet peaks, as observed experimentally. Considering the asymmetric position of the PbSe core in the core-shell geometry, a ratio of $\Gamma_{2}{ }^{\mathrm{h}} / \Gamma_{1}{ }^{\mathrm{h}}=1$ is realistic. The inset in Figure $3 \mathrm{~b}$ shows the relative contributions of electrons and holes to the total current. Since the hole-tunneling rates are much smaller than the electrontunneling rates, the hole contribution to the total tunnel 

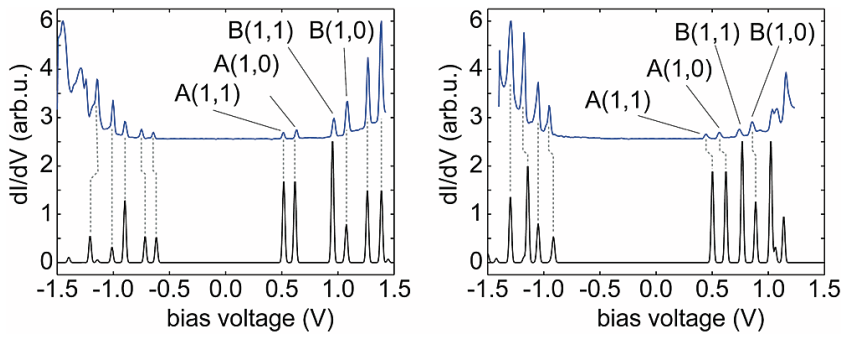

FIGURE 4. Comparison between simulated (black, bottom) and experimental (blue, top) differential conductance spectra of two $\mathrm{PbSe} / \mathrm{CdSe}$ core-shell QDs (left and right panels). The labels $Z(x, y)$ refer to the first (A) and second (B) conduction levels, with the number of electrons and holes involved in the tunneling process indicated by $\mathrm{x}$ and $\mathrm{y}$, respectively.

current is negligible, that is, all resonances are due to electron tunneling.

The doublet features observed for the core-shell QDs appear in the simulations over a wide range of electron and hole tunneling rates. In Figure $3 d$, several simulated differential conductance spectra are shown. For all simulations, the same energy level structure of the QDs was used, as well as the same values of $E_{\mathrm{e}-\mathrm{e}}, \eta$, and $\varepsilon_{\mathrm{F}}$, that is, only the values of the tunneling rates and the recombination rate were changed; the values are given in Table 1. The doublet-peaks are clearly recognizable in all four spectra, demonstrating that the hole-tunneling rates can be varied over 7 orders of magnitude. In addition, including electron-hole recombination does not lead to any qualitative changes in the spectra, as long as the recombination rate is smaller than $\Gamma_{2}{ }^{h}$. If the value becomes larger, the hole occupation of the QD decreases and the hole-induced tunneling resonance becomes less pronounced.

Now, we will reproduce the observed resonances both at negative and positive bias within the hole-induced electron tunneling Ansatz. In Figure 4, experimental spectra of two different QDs and the corresponding simulated spectra are shown. The experimental conductance spectra can be accurately reproduced by the simulations. The master equation simulations allow us to assign specific transitions in the QD to the tunneling resonances (see Figure 4). We did not include electron-hole recombination in these specific simulations due to the long exciton lifetime in PbSe and type II QDs. ${ }^{8,29,30}$ In addition, the peak intensities are influenced by factors that are not included in the model, such as the envelope wave function and the change in barrier height with increasing bias voltage. In addition, because of the small offset of the conduction band of the PbSe core and the CdSe shell, the electronic structure of the unoccupied levels is complicated by hybridization between PbSe and CdSe orbitals. Elucidating the finer details of the electronic structure, including the effect of the CdSe shell on the intervalley splitting in PbSe, will require numerical tight-binding or pseudopotential calculations. The resonances at negative bias are also reproduced by the theory and are due to electron tunneling in the presence of holes. However, the situation is more complicated than at positive bias. The same parameters can be used to reproduce the experimental conductance spectra of several different QDs.

Now, we will turn to the interpretation of the intradoublet peak spacing. The reference energy is taken to be the energy of a neutral QD. The total energy of the QD in the presence of a hole is than given by $E_{\mathrm{QD}, \mathrm{h}}=\varepsilon_{1 \mathrm{~h}}+E_{\mathrm{pol}}{ }_{\mathrm{h}}$, where $\varepsilon_{1 \mathrm{~h}}$ is the energy of the first hole level and $E_{\mathrm{pol}}{ }^{\mathrm{h}}$ is the energy associated with the polarization of the QD due to the presence of a hole. When an electron is injected in the presence of a hole (first peak of the doublet), the total energy becomes: $E_{\mathrm{QD} \text {,eh }}=\varepsilon_{1 \mathrm{~h}}$ $+E_{\text {pol }}{ }^{\mathrm{h}}+\varepsilon_{1 \mathrm{e}}+E_{\mathrm{pol}}^{\mathrm{e}}-E_{\mathrm{e}-\mathrm{h}}$, with $\varepsilon_{1 \mathrm{e}}$ the energy of the first electron level and $E_{\mathrm{e}-\mathrm{h}}$ the electron-hole interaction energy. Hence, the energy that is needed to inject an electron in the presence of a hole is: $E_{1}=\varepsilon_{1 \mathrm{e}}+E_{\mathrm{pol}}^{\mathrm{e}}-E_{\mathrm{e}-\mathrm{h}}$. With similar arguments, the energy required to inject an electron in a neutral QD (second peak of the doublet) is found to be $E_{2}=$ $\varepsilon_{1 \mathrm{e}}+E_{\mathrm{pol}}^{\mathrm{e}}$. Because of the high dielectric constant of PbSe ( $\varepsilon$ $=250$ ), the electric field in the core of the QD is small, and consequently the absolute Stark shift of the energy levels can be estimated to be $<1 \mathrm{meV}$. In addition, possible effects due to a Stark shift of the energy levels only enter through the difference between the Stark shifts at the bias voltages of the electron tunneling and the hole-induced electron tunneling channels. Since this is expected to be significantly smaller than the absolute value of the Stark shift, possible influences of the electric field can be neglected. Therefore, the energy difference, $E_{2}-E_{1}$, that is, the intradoublet peak spacing, gives the electron-hole interaction energy.

By taking into account the bias voltage distribution in the double-barrier tunneling junction $(\eta=0.7-0.8),{ }^{17,22,26}$ we obtain a value of $80 \pm 10 \mathrm{meV}$ for the electron-hole interaction energy in these PbSe-CdSe core-shell QDs. These measurements provide direct experimental information of the electron-hole interaction energy in QDs. The intradoublet peak spacing is the same for all electronic levels (to within a few percent) (see Figure 2b), that is, the electron-hole interaction energy does not dependent on the symmetry of the envelope wave function. We emphasize that electrical spectroscopy is the only way to obtain the electron-hole interaction energy in a direct way.

In summary, we have demonstrated a robust holeinduced electron tunneling mechanism in PbSe-CdSe coreshell QDs. By trapping a positively charged hole in the core of the QD, an additional channel for electron transport opens up at lower applied bias compared to a neutral QD. The difference in the bias voltage between the novel transport channel and the traditional tunneling pathway gives directly the electron-hole interaction energy for each electron level. Our results are important for electrically controlled singlephoton emission. For instance, setting a bias between the peaks of the first doublet will lead to the emission of single photons. The emission rate is controlled by the rate of hole injection into the core of the core-shell QD. Finally, as the two pathways for electron transport are separated in energy 
by $80 \mathrm{meV}$, the observed situation would make wellcontrolled electrically driven single photon emission possible, even at room temperature.

Acknowledgment. We thank Wiel Evers and Celso de Mello Donegá for providing the QDs and Christophe Delerue for discussions. This research was supported by the EU (ITN "HERODOT") and NWO (Chemical Sciences, Vidi-Grant 700.56.423, Rubicon Grant 680.50.0907).

\section{REFERENCES AND NOTES}

(1) Murray, C. B.; Kagan, C. R.; Bawendi, M. G. Synthesis and characterization of monodisperse nanocrystals and closepacked nanocrystal assemblies. Annu. Rev. Mater. Sci. 2000, 30, 545 .

(2) Talapin, D. V.; Lee, J. S.; Kovalenko, M. V.; Shevchenko, E. V. Prospects of Colloidal Nanocrystals for Electronic and Optoelectronic Applications. Chem. Rev. 2010, 110, 389.

(3) de Mello Donegá, C.; Liljeroth, P.; Vanmaekelbergh, D. Physicochemical evaluation of the hot-injection method, a synthesis route for monodisperse nanocrystals. Small 2005, 1, 1152 .

(4) Kim, S.; Fisher, B.; Eisler, H.-J.; Bawendi, M. Type-II Quantum Dots: CdTe/CdSe(Core/Shell) and CdSe/ZnTe(Core/Shell) Heterostructures. J. Am. Chem. Soc. 2003, 125, 11466.

(5) Balet, L. P.; Ivanov, S. A.; Piryatinski, A.; Achermann, M.; Klimov, V. I. Inverted Core/Shell Nanocrystals Continuously Tunable between Type-I and Type-II Localization Regimes. Nano Lett. 2004, 4, 1485

(6) Kudera, S.; Carbone, L.; Casula, M. F.; Cingolani, R.; Falqui, A. Snoeck, E.; Parak, W. J.; Manna, L. Selective Growth of PbSe on One or Both Tips of Colloidal Semiconductor Nanorods. Nano Lett. 2005, 5, 445 .

(7) Milliron, D. J.; Hughes, S. M.; Cui, Y.; Manna, L.; Li, J.; Wang, L.W.; Alivisatos, A. P. Colloidal nanocrystal heterostructures with linear and branched topology. Nature 2004, 430, 190.

(8) Chin, P. T. K.; de Mello Donegá, C.; van Bavel, S. S.; Meskers, S. C. J.; Sommerdijk, N. A. J. M.; Janssen, R. A. J. Highly Luminescent CdTe/CdSe Colloidal Heteronanocrystals with Temperature-Dependent Emission Color. J. Am. Chem. Soc. 2007, 129, 14880.

(9) Klimov, V. I.; Ivanov, S. A.; Nanda, J.; Achermann, M.; Bezel, I.; McGuire, J. A.; Piryatinski, A. Single-exciton optical gain in semiconductor nanocrystals. Nature 2007, 447, 441

(10) Gur, I.; Fromer, N. A.; Geier, M. L.; Alivisatos, A. P. Air-stable allinorganic nanocrystal solar cells processed from solution. Science 2005, 310, 462

(11) Averin, D. V.; Korotkov, A. N.; Likharev, K. K. Theory of singleelectron charging of quantum wells and dots. Phys. Rev. B 1991, 44,6199 .

(12) Likharev, K. K. Single-electron devices and their applications Proc. IEEE 1999, 87, 606.

(13) Mesoscopic Electron Transport, Sohn, L. L.; Kouwenhoven, L. P . Schön, G., Eds.; Kluwer: Dordrecht, 1997.
(14) Delerue, C.; Lannoo, M. Nanostructures; Theory and Modelling; Springer: Berlin, 2004.

(15) Hanson, R.; Kouwenhoven, L. P.; Petta, J. R.; Tarucha, S.; Vandersypen, L. M. K. Spins in few-electron quantum dots. Rev. Mod. Phys. 2007, 79, 1217.

(16) Jdira, L.; Liljeroth, P.; Stoffels, E.; Vanmaekelbergh, D.; Speller, S. Size-dependent single-particle energy levels and interparticle Coulomb interactions in CdSe quantum dots measured by scanning tunneling spectroscopy. Phys. Rev. B 2006, 73, 115305.

(17) Liljeroth, P.; Jdira, L.; Overgaag, K.; Grandidier, B.; Speller, S.; Vanmaekelbergh, D. Can scanning tunnelling spectroscopy measure the density of states of semiconductor quantum dots. Phys. Chem. Chem. Phys. 2006, 8, 3845.

(18) Niquet, Y. M.; Delerue, C.; Allan, G.; Lannoo, M. Interpretation and theory of tunneling experiments on single nanostructures. Phys. Rev. B 2002, 65, 165334

(19) Banin, U.; Cao, Y. W.; Katz, D.; Millo, O. Identification of atomiclike electronic states in indium arsenide nanocrystal quantum dots. Nature 1999, 400, 542.

(20) Banin, U.; Millo, O. Tunneling and optical spectroscopy of semiconductor nanocrystals. Annu. Rev. Phys. Chem. 2003, 54, 465.

(21) Liljeroth, P.; Overgaag, K.; Urbieta, A.; Grandidier, B.; Hickey, S. G.; Vanmaekelbergh, D. Variable orbital coupling in a twodimensional quantum-dot solid probed on a local scale. Phys. Rev. Lett. 2006, 97, 096803.

(22) Overgaag, K.; Liljeroth, P.; Grandidier, B.; Vanmaekelbergh, D. Scanning tunneling spectroscopy of individual PbSe quantum dots and molecular aggregates stabilized in an inert nanocrystal matrix. ACS Nano 2008, 2, 600.

(23) Steiner, D.; Dorfs, D.; Banin, U.; Della Sala, F.; Manna, L.; Millo, O. Determination of band offsets in heterostructured colloidal nanorods using scanning tunneling spectroscopy. Nano Lett. 2008, 8, 2954.

(24) Pietryga, J. M.; Werder, D. J.; Williams, D. J.; Casson, J. L.; Schaller, R. D.; Klimov, V. I. Utilizing the Lability of Lead Selenide to Produce Heterostructured Nanocrystals with Bright, Stable Infrared Emission. J. Am. Chem. Soc. 2008, 130, 4879.

(25) Lambert, K.; Geyter, B. D.; Moreels, I.; Hens, Z. PbTe|CdTe CorellShell Particles by Cation Exchange, a HR-TEM study. Chem. Mater. 2009, 21, 778 .

(26) Sun, Z.; Swart, I.; Delerue, C.; Vanmaekelbergh, D.; Liljeroth, P. Orbital and Charge-Resolved Polaron States in CdSe Dots and Rods Probed by Scanning Tunneling Spectroscopy. Phys. Rev. Lett. 2009, 102, 196401.

(27) Liljeroth, P.; Zeijlmans van Emmichoven, P. A.; Hickey, S. G.; Weller, H.; Grandidier, B.; Allan, G.; Vanmaekelbergh, D. Density of states measured by scanning-tunneling spectroscopy sheds new light on the optical transitions in PbSe nanocrystals. Phys. Rev. Lett. 2005, 95, No. 086801

(28) Franceschetti, A.; Williamson, A.; Zunger, A. Addition Spectra of Quantum Dots: the Role of Dielectric Mismatch.J. Phys. Chem. B $2000,104,3398$

(29) Allan, G.; Delerue, C. Confinement effects in PbSe quantum wells and nanocrystals. Phys. Rev. B 2004, 70, 245321.

(30) An, J. M.; Franceschetti, A.; Zunger, A. The excitonic exchange splitting and radiative lifetime in PbSe quantum dots. Nano Lett. 2007, 7, 2129 